\title{
Dosimetric impact of intrafraction prostate rotation and accuracy of gating, multi-leaf collimator tracking and couch tracking to manage rotation: an end-to-end validation using volumetric film measurements
}

Robin De Roover ${ }^{1,2,}{ }^{*}$, Rune Hansen ${ }^{3}$, Wouter Crijns ${ }^{1,2}$, Casper Gammelmark Muurholm ${ }^{4}$, Kenneth Poels $^{1,2}$, Simon Skouboe ${ }^{4}$, Karin Haustermans ${ }^{1,2}$, Per Rugaard Poulsen ${ }^{4,5}$ and Tom Depuydt ${ }^{1,2,{ }^{*}}$

\begin{tabular}{l|l}
\hline Robin De Roover & robin., deroover@kuleuven.be \\
\hline Rune Hansen $^{3}$ & rune.hansen@aarhus.rm.dk \\
\hline Wouter Crijns $^{1,2}$ & wouter.crijns@kuleuven.be \\
\hline Casper Gammelmark Muurholm & casmuu@rm.dk \\
\hline Kenneth Poels ${ }^{1,2}$ & kenneth.poels@uzleuven.be \\
\hline Simon Skouboe & simsko@rm.dk \\
\hline Karin Haustermans & karin.haustermans@kuleuven.be \\
\hline Per Rugaard Poulsen & per.poulsen@rm.dk \\
\hline Tom Depuydt ${ }^{1,2, *}$ & tom.depuydt@kuleuven.be \\
\hline
\end{tabular}

${ }^{1}$ Department of Oncology, KU Leuven 3000 Leuven, Belgium

${ }^{2}$ Department of Radiation Oncology, University Hospitals Leuven, 3000 Leuven, Belgium

${ }^{3}$ Department of Medical Physics, Aarhus University Hospital, 8200 Aarhus N, Denmark

${ }^{4}$ Department of Oncology, Aarhus University Hospital, 8200 Aarhus N, Denmark

${ }^{5}$ Danish Center for Particle Therapy, Aarhus University Hospital, 8200 Aarhus N, Denmark

${ }^{*}$ Corresponding authors:

Robin De Roover; robin.deroover@uzleuven.be;

Tom Depuydt; tom.depuydt@uzleuven.be;

Laboratory of Experimental Radiotherapy, Department of Oncology, KU Leuven

UZ Herestraat $49-$ box 7003

3000 Leuven, Belgium.

Short running title: Dosimetric impact of prostate rotations

Keywords: Prostate cancer, Stereotactic body radiotherapy, Focal boost, Gating, Couch tracking, MLC tracking

Bullet points:

- Intrafraction prostate rotations reduces target coverage and increases OAR dose.

- Gating, MLC tracking and couch tracking improved the dosimetric accuracy.

- Residual motion-related dose errors remained due to the lack of rotation correction.

Conflicts of interest: This work was partly supported by Varian Medical Systems. 


\section{Abstract}

Background and purpose: Both gating and tracking can mitigate the deteriorating dosimetric impact of intrafraction translation during prostate stereotactic body radiotherapy (SBRT). However, their ability to manage intrafraction rotation has not yet been thoroughly investigated. The dosimetric accuracy of gating, MLC tracking and couch tracking to manage intrafraction prostate rotation was investigated.

Materials and methods: Treatment plans for end-to-end tests of prostate SBRT with focal boosting were generated for a dynamic anthropomorphic pelvis phantom. The phantom applied internal lateral rotation (up to $25^{\circ}$ ) and coupled vertical and longitudinal translation of a radiochromic film stack that was used for dose measurements. Dose was delivered for each plan while the phantom applied motion according to three typical prostate motion traces without compensation (i), with gating (ii), with MLC tracking (iii) or with couch tracking (iv). Measured doses for the four motion compensation strategies were compared with the planned dose in terms of $y$-index analysis, target coverage and organs at risk (OAR) sparing.

Results: Intrafraction rotation reduced the $3 \%($ global $) / 2 m m ~ \gamma$-index passing rate $(\gamma \mathrm{PR})$ for the prostate target volume by median (range) $-33.2 \%(-68.6 \%,-4.1 \%)$ when no motion compensation was applied. The use of motion compensation improved the $\gamma$ PR by $13.2 \%(-0.4 \%, 32.9 \%)$ for gating, by $6.0 \%(-0.8 \%$, $27.7 \%)$ for MLC tracking and by $11.1 \%(1.2 \%, 22.9 \%)$ for couch tracking. The three compensation techniques improved the target coverage in most cases. Gating showed better OAR sparing than MLC tracking or couch tracking.

Conclusions: Compensation of intrafraction prostate rotation with gating, MLC tracking and couch tracking was investigated experimentally for the first time. All three techniques improved the dosimetric accuracy, but residual motion-related dose errors remained due to the lack of rotation correction. 


\section{Introduction}

Stereotactic body radiotherapy (SBRT) is increasingly being used in the treatment of localized prostate cancer (PCa) as ultrahypofractionated treatments have been demonstrated to provide good disease control with minimal toxicity and are more convenient for the patient [1-7]. However, due to the high dose-per-fraction delivered by such ultrahypofractionated treatments, there is a need for steep dose gradients to be employed with tighter than conventional margins as to limit exposure of surrounding organs at risk (OAR) $[8,9]$. Hence, there is an increased need to minimize geometric uncertainty. The mobility of the prostate is known as a potential source of uncertainty during treatment delivery showing both persistent drifts and sudden transient displacements [10-12]. The use of gating or tumour tracking has been shown to mitigate the dosimetric impact of intrafraction translations improving treatment accuracy [13-15]. Gating monitors the target position during treatment and pause the delivery if the motion exceeds a preset threshold after which a treatment couch correction can be performed to correct for persistent displacements [16-19]. Tumour tracking, on the other hand, continuously adapts the delivery to the target motion by adapting the multi-leaf collimator (MLC tracking) or by counter-movement of the target using the treatment couch (couch tracking) [20-22]. The dosimetric accuracy of these techniques has been investigated extensively through dynamic phantoms measurements, often using detector arrays mounted on a motion stage to apply intrafraction translations [23-29].

However, besides translations, intrafraction rotations occur with the prostate undergoing a rotation of more than $5^{\circ}$ for about a third of the total treatment [30]. Moreover, lateral rotations up to $25^{\circ}$ have been observed [31]. The dosimetric impact of such intrafraction rotations have been investigated insilico but these studies often use the static average rotation and show conflicting results [32-34]. In addition, the ability of gating and tumour tracking to manage rotation has not yet been thoroughly investigated. 
In this study, the impact of intrafraction prostate rotation and the accuracy of gating, MLC tracking and couch tracking to manage rotation was investigated using a dynamic pelvis phantom that applied internal rotation and translation of an inserted film stack.

\section{Materials and methods}

\section{The phantom and mapped patient anatomy}

An anthropomorphic dynamic pelvis phantom (CIRS, Norfolk, VA, USA) was used for treatment planning, internal motion application and dose measurements (Figure 1A). The phantom is tissue equivalent and contains an anthropomorphic pelvis with cortical and trabecular bone. One side of the phantom houses a cavity for insertion of a rotatable disk containing an off-centre cubic cut-out to hold the film stack. The rotatable disk allowed for applying internal lateral rotation in the sagittal plane. Rotation of the disk was driven by longitudinal translation of a driving rod connected to an external linear actuator that can reproduce the programmed motion with sub-mm accuracy (Figure $1 \mathrm{~A})$. The resulting lateral rotations were reproduced with an accuracy of $<1^{\circ}$. In neutral position, the film stack was rotated by a $47^{\circ}$ lateral rotation with respect to the longitudinal axis.

Delineated anatomical structures of two men with prostate cancer and MRI-visible tumour who were previously treated on the FLAME trial (NCT01168479) [35] were rigidly mapped onto the phantom to correspond with the film stack (Figure 1B). For both patients, CT and registered multiparametric MRI (mpMRI) image sequences were available. The whole prostate gland was delineated as target volume [36] and the intraprostatic lesion(s) visible on $\mathrm{mpMRI}$ were delineated as focal boost volume (GTV $\mathrm{boost}_{\text {) }}$ $[37,38]$. The prostate CTV ( $\left.\mathrm{CTV}_{\text {prostate }}\right)$ included the whole prostate and a $4 \mathrm{~mm}$ isotropic margin around GTV boost. A second seminal vesicle CTV (CTV SV) was created for both patients [39]. Rectum, bladder and urethra were delineated as OAR [40]. The entire $\mathrm{CTV}_{\text {prostate }}$ with $\mathrm{GTV}_{\text {boost }}$ and intraprostatic urethra were located within the film stack whereas the adjacent rectum and bladder were only partially included (Figure 1B). The apex of the mapped prostate contour was located near the lateral rotation axis of the 
translation of the prostate centroid in the sagittal plane as shown in Figure 1C.

Three Calypso radiofrequency transponders (Varian Medical Systems, Palo Alto, CA, USA) were embedded into the film stack, one at the apex and two at the base of the mapped prostate, for monitoring of the applied motion. The Calypso centroid was located near the centroid of the mapped

centroid.
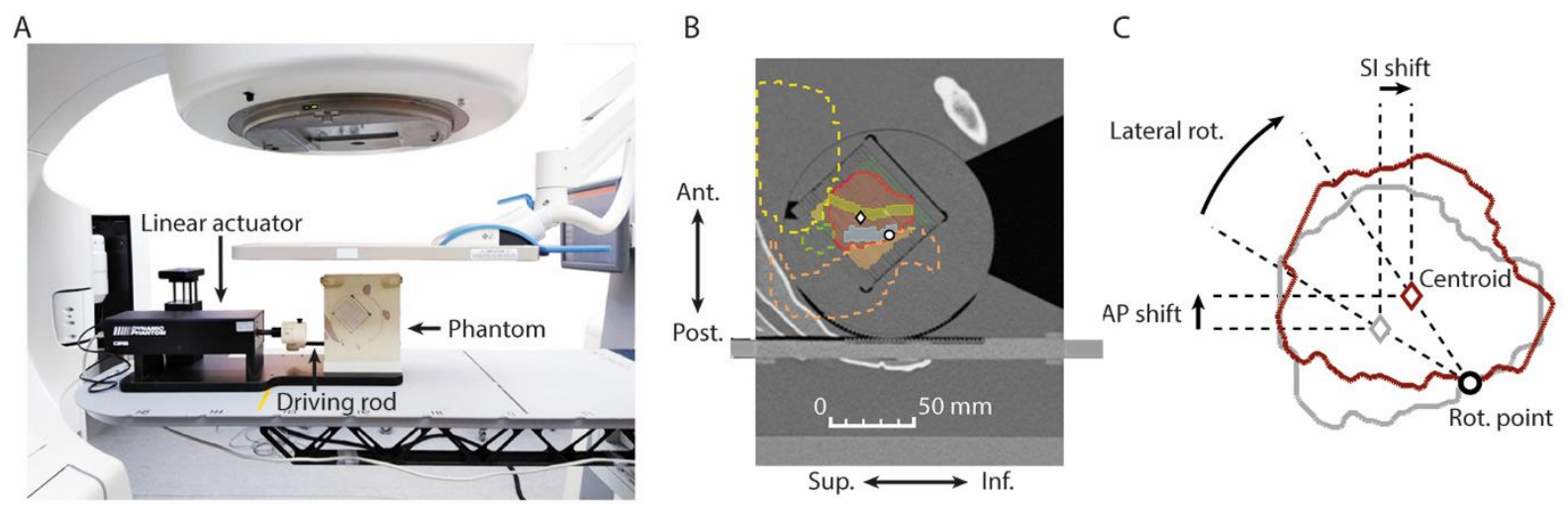

Figure 1. Overview of experimental setup and phantom geometry. (A) Experimental setup used for MLC tracking and couch tracking measurements. The gating measurements were performed without the Calypso antenna as these used the on-board kV imager for motion monitoring. (B) Sagittal CT slice displaying the pelvis anatomy of the anthropomorphic phantom together with the mapped contours of a patient case (prostate: red, GTV boost: blue, urethra and bladder: yellow, rectum: brown). The shaded area shows the radiochromic film stack used for dose measurements. The rotation point (circle) of the rotatable disk and the prostate centroid (diamond) are annotated. (C) Schematic illustration of how lateral rotation (rot.) around the rotation point causes a coupled vertical (AP) and longitudinal (SI) shift of the prostate centroid in the sagittal plane. The mapped prostate contour of (B) is shown in its neutral position (grey contour) and after applying an anterior lateral rotation of $25^{\circ}$ around the rotation point (red contour), which is located near the apex.

\section{Treatment planning}

Treatment plans for end-to-end tests of prostate SBRT with focal boosting were generated for the pelvis phantom using the delineated anatomical structures that were rigidly mapped onto a CT scan of the phantom (Figure 1B). Target volume dose prescriptions and OAR dose constraints were based on the hypo-FLAME trial (NCT02853110) [41,42] and are available in Supplementary Table 1 . The prescribed dose was $35 \mathrm{~Gy}$ to $\mathrm{CTV}_{\text {prostate }}$ and $30 \mathrm{~Gy}$ to $\mathrm{CTV}_{\mathrm{SV}}$ delivered in five fractions. The objectives

for $\mathrm{GTV}_{\text {boost }}$ aimed to deliver a minimal total dose of $40 \mathrm{~Gy}$ on at least $99 \%$ of the volume and up to 50

Gy as long as the OAR sparing was not at risk. The rectum and urethra maximum dose $\left(D_{0.035 c c}\right)$ were 
restricted to $40 \mathrm{~Gy}$. Planning target volumes (PTV) were created by expanding the CTVs using an isotropic $4 \mathrm{~mm}$ margin $[41,43]$.

Treatment planning was performed in Eclipse version 15.6 treatment planning system (TPS) (Varian

Medical Systems) using the photon optimizer (PO) algorithm version 15.6 and the analytical anisotropic algorithm (AAA) version 15.6 .03 at $1 \mathrm{~mm}$ grid size for dose calculation. The planning technique consisted of $6 \mathrm{MV}$ dual-arc volumetric modulated arc therapy (VMAT) with collimator rotations set at $10^{\circ}$ and $80^{\circ}$ [41]. An avoidance sector between $170^{\circ}$ and $190^{\circ}$ gantry angle was used for both arcs to limit entrance dose to the rectum. Plans were normalized such that 35 Gy covered $95 \%$ of PTV prostate.

\section{Motion traces}

Three typical prostate motion traces recorded by Langen et al. [11] were selected for this study. The recorded traces only included 3D translations of the prostate centroid and contained no rotations. However, several studies of interfraction prostate motion showed that motion in the sagittal plane can be approximated by lateral rotation around the apex $[44,45]$. Therefore, the lateral rotation applied by the phantom was derived by converting the recorded 2D sagittal translations of the prostate centroid into lateral rotations around the rotation point as shown in Figure $1 \mathrm{C}$. The used sections of the derived traces are shown in Figure 2 together with a boxplot of the resulting centroid shift and the temporal displacement fraction during dose delivery. 
A

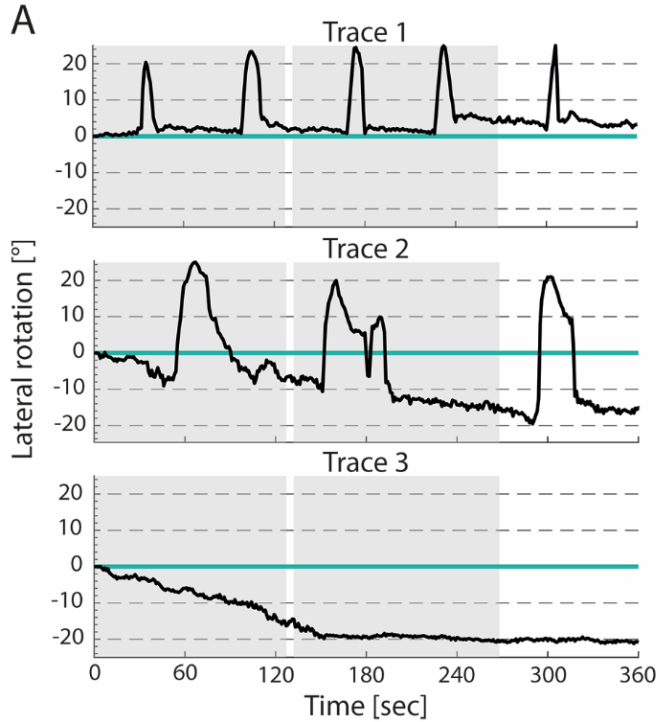

B

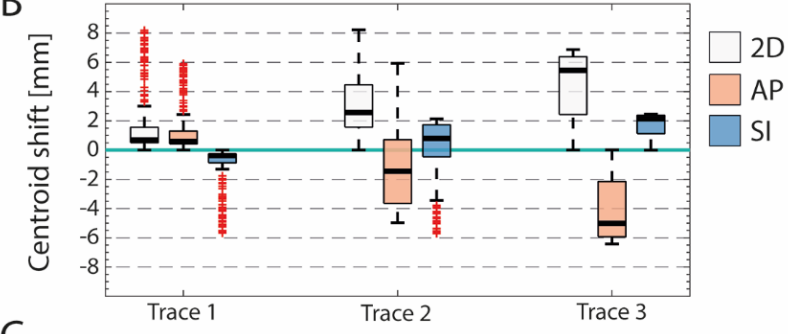

C

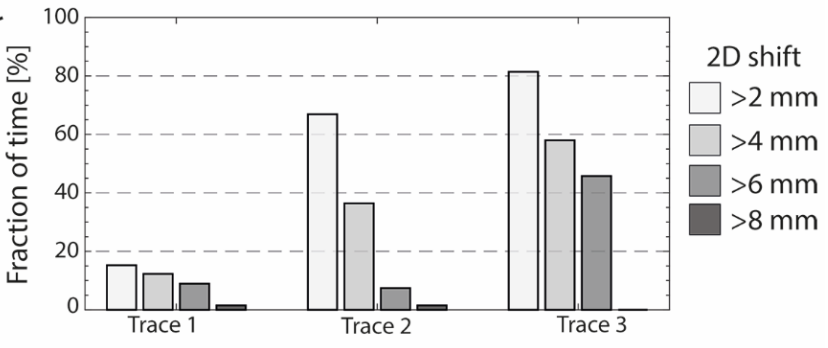

Figure 2. Applied motion traces in this study. (A) Internal lateral rotation of the motion traces applied by the phantom during dynamic measurements: high frequency anterior excursions (Trace 1), a posterior drift with anterior jumps (Trace 2) and a rapid posterior drift (Trace 3). The shaded area denotes the time at which the dose is delivered. (B) Boxplot of the 2D, vertical (AP) and longitudinal (SI) centroid shift in the sagittal plane due to the applied lateral rotation during dose delivery. (C) Temporal fraction of the 2D centroid shift larger than 2, 4,6 and $8 \mathrm{~mm}$ during dose delivery.

\section{Treatment delivery}

Treatments were delivered to the dynamic pelvis phantom using two TrueBeam version 2.7 linear accelerators equipped with Millennium MLC (Varian Medical Systems). Gating experiments were performed on a TrueBeam equipped with PerfectPitch treatment couch whereas tracking experiments used the standard treatment couch. Prior to each delivery, the film stack was positioned in neutral position and the target position was verified through orthogonal kV imaging and a marker-based 3D couch correction by using the Calypso transponders as fiducial markers. Subsequently, the dose was delivered for each plan while the phantom applied internal motion according to the three traces and using four motion compensation strategies: no compensation, gating, MLC tracking or couch tracking. In total, six cases (two treatment plans and three motion traces) were measured for each compensation strategy. An additional static delivery of each plan was measured for both linacs as benchmark. For each measurement, one fraction of the plan was delivered.

Gating used Triggered Imaging with Auto Beam Hold (Varian Medical Systems) in Treatment Mode to capture a $\mathrm{kV}$ image every $3 \mathrm{~s}$ and pause the delivery if one of the transponders was detected in the 
image outside a $4 \mathrm{~mm}$ tolerance region, which corresponds to the CTV-to-PTV margin. A Calypso transponder-specific template was used for automated detection of the transponders in the image. Orthogonal kV imaging and a 6 degrees-of-freedom (6DoF) couch correction was performed if the displacement was persistent in subsequent $\mathrm{kV}$ images. The maximum lateral rotation correction of the couch was limited to $3^{\circ}[46]$.

Tracking was performed using the iTools Tracking platform (Varian Medical Systems) in TrueBeam

Developer Mode. The real-time 3D positions of the three radiofrequency transponders embedded in the film stack were monitored with the Calypso system (Varian Medial Systems) and the corresponding 3D target centroid position was transferred to the accelerator at a rate of $25 \mathrm{~Hz}$. A linear Kalman prediction filter was applied to the signal to partially compensate for tracking system latencies [47]. The predicted target centroid translation was then compensated by adapting the treatment field via the MLC or by counter-movement of the target using the treatment couch. The same tracking system was previously described by Hansen et al. for MLC and couch tracking [27].

\section{Volumetric film measurements}

The dose delivered by each treatment scenario was measured using a radiochromic film stack inserted into the phantom allowing 2D film measurements in 21 planes separated by $2 \mathrm{~mm}$ thick layers of polystyrene. Each plane contained a square piece $\left(63 \times 63 \mathrm{~mm}^{2}\right)$ of GafChromic EBT3 film (Ashland Specialty Ingredients, Wayne, NJ, USA) that was laser-cut to fit in the film stack. The relative orientation of the film planes with respect to the mapped contours is illustrated in Supplementary Figure 1.

After dose delivery, the film pieces were reconstructed in their original order to be scanned using an Epson 12000XL Pro flatbed scanner (Seiko Epson Corporation, Japan) at $0.169 \mathrm{~mm}$ resolution. The transmittance was converted to dose in MeVisLab version 2.5 (MeVis Medical Solutions AG, Bremen, Germany) through the calibration method of Crijns et al., which uses two page sized calibration films to estimate the dose response function [48]. The lateral scan effect was resolved in the calibration function itself [48] whereas the three colour channels of each scan were combined to perform 
multichannel nonuniformity correction [49]. To correct for daily output variation of the linear accelerator and the scan-to-scan and film-to-film response variability, a reference film piece was homogeneously irradiated to a dose of $7 \mathrm{~Gy}$ after each measurement and included during scanning [50].

Next, the 2D dose images were sorted into the 21 different film planes. Each film plane was rigidly aligned with a template using the assembling guiding holes for the film stack as landmarks. The aligned film planes were stacked using a plane separation of $2.5 \mathrm{~mm}$, which accounts for the $2 \mathrm{~mm}$ separation between consecutive films and the film thickness. The stacked dose planes were then resampled into 20 dose slices by assigning the dose in each voxel to the average value of its corresponding pixel in the lower and upper film plane. A 2D median and Wiener filter over $6 \times 6$ pixels $\left(1.02 \times 1.02 \mathrm{~mm}^{2}\right)$ was applied to each slice to reduce noise. The resulting measured volumetric dose distribution consisted of 20 slices with $0.169 \mathrm{~mm}$ in-plane resolution and $2.5 \mathrm{~mm}$ slice thickness.

\section{Dosimetric performance}

The dosimetric performance of the four compensation strategies was evaluated by comparing the dose measurements with the planned dose. Prior to comparison, the smooth TPS dose distributions (1 mm grid size) were resampled to the film stack's coordinate system using a tri-linear interpolation in SlicerRT [51]. As such, all comparisons were based on dose distributions with an identical coordinate system and resolution.

The accuracy between measured and planned dose was evaluated using $2 \mathrm{D} 3 \% / 2 \mathrm{~mm} \gamma$-index analysis

$165[52,53]$ for the target structures ( $\mathrm{CTV}_{\text {prostate }}$ and $\left.\mathrm{GTV}_{\text {boost }}\right)$ and the OARs (rectum, bladder and urethra) as recommended by the AAPM TG-218 report [54]. The dose differences were normalized globally to the prescribed dose level of $7 \mathrm{~Gy}$ for the $\mathrm{CTV}_{\text {prostate }}$ and only doses above $10 \%$ were included in the evaluation. For the target structures, the volume receiving the prescribed dose ( $\mathrm{CTV}_{\text {prostate }} \mathrm{V}_{7 \mathrm{~Gy}}$ and GTV $V_{\text {boost }} \mathrm{V}_{8 \mathrm{G} y}$ ) was determined to assess coverage. In addition, changes in the dose to $95 \%$ of the volume $\left(\Delta D_{95 \%}\right)$ and the mean dose $\left(\Delta D_{\text {mean }}\right)$ were evaluated. For the OARs, the change in the maximum dose 
$\left(\Delta D_{0.035 c c}\right)$ was evaluated. The $\gamma$-index passing rate $(\gamma P R)$ and changes in dose-volume parameters for the different scenarios were compared using a Kruskal-Wallis omnibus test followed by two-sided Wilcoxon's matched-pairs signed-rank tests. Statistical significance was determined using the Benjamini-Hochberg procedure to correct for multiple comparisons by controlling the false discovery rate at significance level 0.05 [55]. All evaluations and statistical tests were performed in Matlab R2017b (Mathworks, Natick, MA, USA).

\section{Results}

Static film measurements showed good dosimetric agreement with respect to the planned dose with a median 3\%(global)/2mm $\mathrm{PPR}$ over the $\mathrm{CTV}_{\text {prostate }}$ of $95.0 \%$ (range: $88.6 \%, 99.6 \%$ ). The application of intrafraction lateral rotation decreased the $\mathrm{PPR}$ over the $\mathrm{CTV}_{\text {prostate }}$ for all cases by median $-33.2 \%$ (range: $-68.6 \%,-4.1 \%$ ) compared to the static measurements when no motion compensation was applied (Table 1). The use of motion compensation improved the YPR over the $\mathrm{CTV}_{\text {prostate }}$ by $13.2 \%$ ($0.4 \%, 32.9 \%)$ for gating, by $6.0 \%(-0.8 \%, 27.7 \%)$ for MLC tracking and by $11.1 \%(1.2 \%, 22.9 \%)$ for couch tracking compared to no compensation. Despite the observed improvements, residual motion-related dose errors remained when comparing the $\mathrm{PPR}$ to those obtained for the static measurements (Figure 3).

Intrafraction rotations reduced the volume receiving the prescribed dose of both $\mathrm{CTV}_{\text {prostate }}\left(\Delta \mathrm{V}_{7 \mathrm{~Gy}}\right.$ of $0.8 \%(-10.7 \%, 0.9 \%))$ and $\operatorname{GTV}_{\text {boost }}\left(\Delta \mathrm{V}_{8 \mathrm{~Gy}}\right.$ of $\left.-1.5 \%(-19.2 \%, 2.1 \%)\right)$ compared to the static measurements if no motion compensation was used during dose delivery. The use of motion compensation improved the GTV boost $V_{8 G y}$ in 5 out of 6 cases for gating, in 4 out of 6 cases for MLC tracking and in 3 out of 6 cases for couch tracking (Table 1). As such, the $\mathrm{GTV}_{\text {boost }}$ volume receiving $8 \mathrm{~Gy}$ was restored to $\mathrm{V}_{8 \mathrm{~Gy}} \geq$ 95.2\% (gating), $\mathrm{V}_{8 G y} \geq 94.9 \%$ (MLC tracking) and $\mathrm{V}_{8 G \mathrm{y}} \geq 96.7 \%$ (couch tracking), which are close to $\mathrm{V}_{8 G \mathrm{y}}$ $\geq 96.8 \%$ obtained for the static measurements. The CTV $\mathrm{V}_{\text {prostate }} \mathrm{V}_{7 \mathrm{~Gy}}$ was improved in 3 out of 6 cases independent of the used compensation technique (Table 1) and showed residual areas that did not receive the prescribed dose of $7 \mathrm{~Gy}$, i.e., $\mathrm{V}_{7 \mathrm{~Gy}} \geq 90.5 \%$ (gating), $\mathrm{V}_{7 \mathrm{~Gy}} \geq 93.9 \%$ (MLC tracking) and $\mathrm{V}_{7 \mathrm{~Gy}} \geq$ 
94.4\% (couch tracking) compared to $V_{7 G y} \geq 98.4 \%$ for the static measurements (Figure 3). In general, trace 3 (rapid posterior drift) had the largest dosimetric impact on the target coverage without motion compensation and showed the largest improvement when using motion compensation, independent of the used technique (Figure 4).

Depending on the relative location of the OAR and the direction of the applied intrafraction rotation, an increase in $D_{0.035 c c}$ was observed for the rectum $\left(\Delta D_{0.035 c c} \leq+6.3 \%\right)$, the bladder $\left(\Delta D_{0.035 c c} \leq+5.9 \%\right)$ and the urethra $\left(\Delta \mathrm{D}_{0.035 c \mathrm{c}} \leq+10.0 \%\right)$ when no compensation was applied (Table 1$)$. As a consequence, the maximum dose constraint to the urethra of $D_{0.035 c c} \leq 8.4 \mathrm{~Gy}$ was violated in 3 out of 6 cases. The use of gating restored the urethra $D_{0.035 c c}$ to within the clinical threshold of $D_{0.035 c c} \leq 8.4 \mathrm{~Gy}$ for all cases. Both MLC tracking and couch tracking had respectively 2 out of 6 cases and 3 out of 6 cases that violated the urethra $\mathrm{D}_{0.035 c \mathrm{c}} \leq 8.4 \mathrm{~Gy}$ constraint. In general, MLC tracking and couch tracking showed less OAR sparing compared to gating.

More details on the structure specific $3 \%($ global)/2mm $\gamma \mathrm{PR}$, target coverage and dose-volume parameters are shown in Figure 3. The corresponding values are given in Supplementary Table 2. The structure specific dosimetric comparison between the different compensation strategies are presented in Table 1. None of the observed differences were statistically significant when correcting for multiple comparisons. A sagittal and axial slice of the $y$-index map together with the dose volume histogram when applying a rapid posterior drift (Trace 3 ) during dose delivery without compensation and with gating, MLC tracking and couch tracking is visualized in Figure 4. 

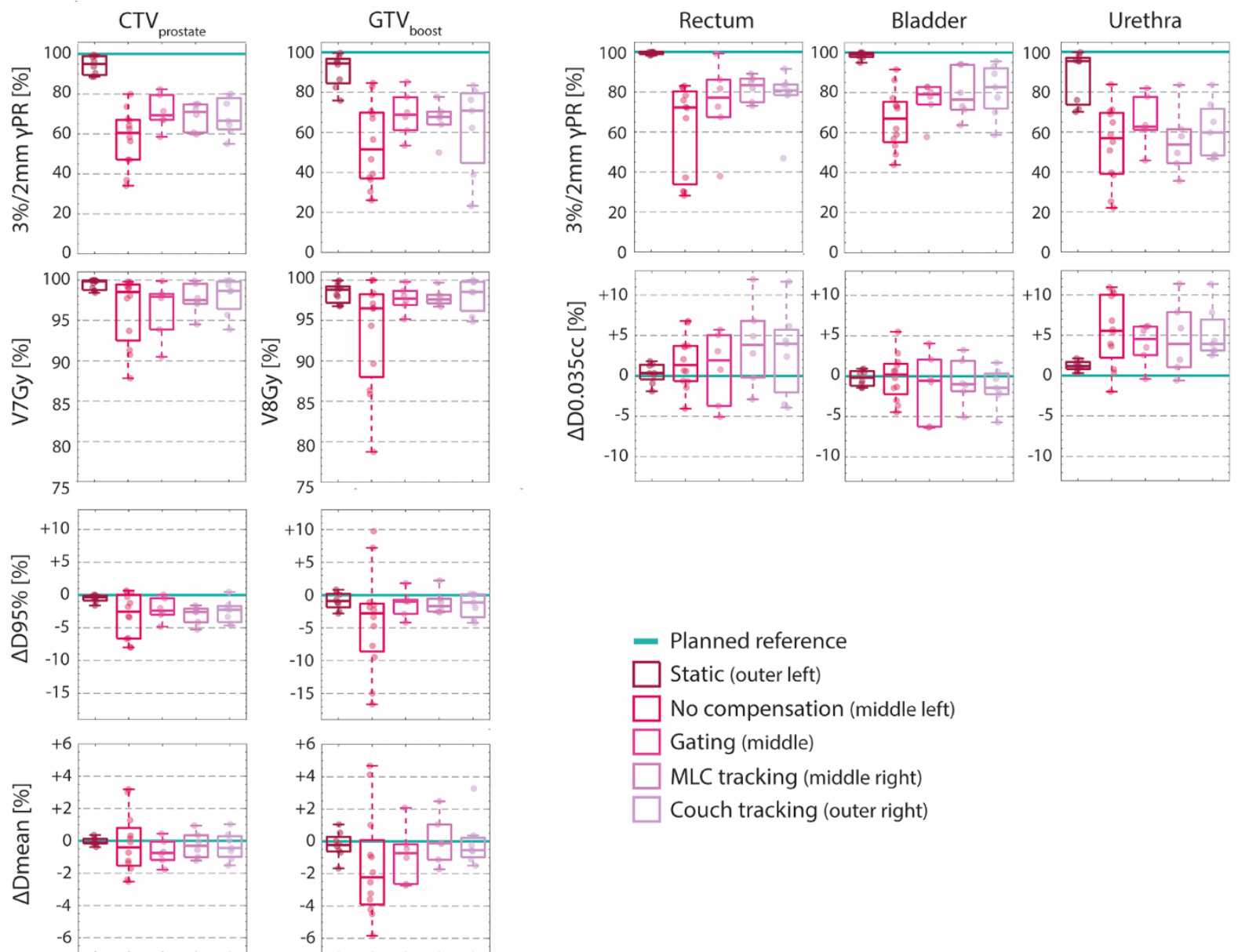

Figure 3. Structure specific dosimetric evaluation. Results are shown as boxplots over both patient cases and all three motion traces combined for the static measurement (outer left in each plot) and the four compensation strategies: no compensation (middle left), gating (middle), MLC tracking (middle right) and couch tracking (outer right). The $3 \%($ global) $2 \mathrm{~mm} \psi$-index passing rates $(\gamma \mathrm{PR})$ are shown for all structures together with the volume receiving the prescribed dose ( $C_{T V} V_{\text {prostate }} \mathrm{V}_{7 \mathrm{~Gy}}$ and $\left.\mathrm{GTV}_{\text {boost }} \mathrm{V}_{8 \mathrm{~Gy}}\right)$, changes in the dose to $95 \%$ of the volume $\left(\triangle \mathrm{D}_{95 \%}\right)$, the mean dose $\left(\Delta D_{\text {mean }}\right)$ for the target structures ( $C T V_{\text {prostate }}$ and $\left.G T V_{\text {boost }}\right)$, and changes in the maximum dose $\left(\Delta \mathrm{D}_{0.035 c c}\right)$ for the organs at risk (rectum, bladder and urethra). The change is with the planned dose as reference. 
Table 1. Structure specific dosimetric comparison. Median and range ( $\mathrm{min}, \mathrm{max}$ ) of the pairwise difference of the $3 \%($ global) $/ 2 \mathrm{~mm} \gamma$-index passing rates $(\gamma \mathrm{PR})$ and dose-volume parameters are given for each structure. None of the observed differences were statistically significant when correcting for multiple comparisons.

\begin{tabular}{|c|c|c|c|c|}
\hline $\begin{array}{l}\text { Evaluation } \\
\text { parameter }\end{array}$ & $\begin{array}{l}\text { No compensation vs } \\
\text { Static } \\
\text { Median (Min, Max) }\end{array}$ & $\begin{array}{c}\text { Gating vs No } \\
\text { compensation } \\
\text { Median (Min, Max) }\end{array}$ & $\begin{array}{l}\text { MLC tracking vs } \\
\text { No compensation } \\
\text { Median (Min, Max) }\end{array}$ & $\begin{array}{l}\text { Couch tracking vs } \\
\text { No compensation } \\
\text { Median (Min; Max) }\end{array}$ \\
\hline \multicolumn{5}{|l|}{ CTV $_{\text {prost }}$} \\
\hline $3 \% / 2 \mathrm{~mm}$ pPR [\%] & $-33.2(-59.4,-15.0)$ & $13.2(-0.4,32.9)$ & $6.0(-0.8,27.7)$ & $11.1(1.2,22.9)$ \\
\hline$\Delta \mathrm{V}_{7 \mathrm{~Gy}}[\%]$ & $-0.8(-10.7,0.9)$ & $0.5(-3.8,7.0)$ & $0.0(-3.6,4.9)$ & $0.0(-4.8,5.7)$ \\
\hline$\Delta \mathrm{D}_{95 \%}[\%]$ & $-2.3(-7.7,2.0)$ & $0.8(-2.7,5.1)$ & $0.5(-4.0,3.3)$ & $0.5(-4.8,3.9)$ \\
\hline$\Delta \mathrm{D}_{\text {mean }}[\%]$ & $-0.4(-2.6,3.1)$ & $0.0(-3.1,3.0)$ & $0.0(-2.8,0.2)$ & $-0.7(-3.7,2.7)$ \\
\hline \multicolumn{5}{|l|}{ GTV boost } \\
\hline $3 \% / 2 \mathrm{~mm}$ pPR [\%] & $-38.5(-68.6,-4.1)$ & $22.9(2.5,28.4)$ & $-2.9(-9.0,29.7)$ & $10.5(-16.4,33.6)$ \\
\hline$\Delta \mathrm{V}_{8 \mathrm{~Gy}}[\%]$ & $-1.5(-19.2,2.1)$ & $2.3(-0.2,18.7)$ & $0.9(-1.7,8.8)$ & $-0.1(-1.0,10.4)$ \\
\hline$\Delta \mathrm{D}_{95 \%}[\%]$ & $-1.7(-16.1,10.2)$ & $2.2(-7.9,15.7)$ & $1.4(-8.3,11.2)$ & $0.0(-5.0,13.9)$ \\
\hline$\Delta \mathrm{D}_{\text {mean }}[\%]$ & $-2.1(-6.0,4.1)$ & $1.2(-2.1,5.7)$ & $1.6(-2.5,3.9)$ & $0.8(-3.6,7.0)$ \\
\hline \multicolumn{5}{|l|}{ Rectum } \\
\hline $3 \% / 2 \mathrm{~mm}$ pPR [\%] & $-27.5(-71.0,-16.0)$ & $11.4(-8.4,44.5)$ & $6.1(0.2,41.1)$ & $8.0(4.3,42.7)$ \\
\hline$\Delta \mathrm{D}_{0.035 \mathrm{cc}}[\%]$ & $0.5(-3.1,6.3)$ & $-1.7(-4.5,9.1)$ & $0.3(-4.1,5.8)$ & $0.6(-3.5,6.4)$ \\
\hline \multicolumn{5}{|l|}{ Bladder } \\
\hline $3 \% / 2 \mathrm{~mm}$ pPR [\%] & $-31.2(-54.8,-7.2)$ & $12.3(-13.5,21.2)$ & $12.8(-8.7,29.3)$ & $15.5(-6.3,20.7)$ \\
\hline$\Delta \mathrm{D}_{0.035 \mathrm{cc}}[\%]$ & $0.0(-4.2,5.9)$ & $-0.9(-6.2,3.4)$ & $-1.2(-5.4,11)$ & $-0.5(-6.6,3.2)$ \\
\hline \multicolumn{5}{|l|}{ Urethra } \\
\hline $3 \% / 2 \mathrm{~mm}$ pPR [\%] & $-28.7(-61.3,-11.6)$ & $9.7(-8.3,23.6)$ & $8.6(-10.4,23.4)$ & $2.6(-3.4,12.3)$ \\
\hline$\Delta \mathrm{D}_{0.035 \mathrm{cc}}[\%]$ & $4.5(-3.1,10.0)$ & $-0.2(-7.4,2.2)$ & $-1.7(-6.7,6.3)$ & $-2.8(-10.5,7.8)$ \\
\hline
\end{tabular}



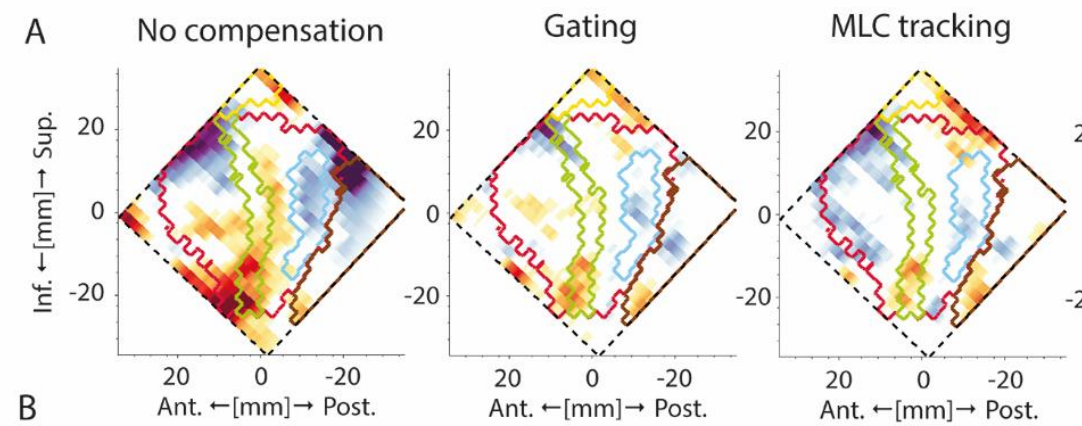

Couch tracking
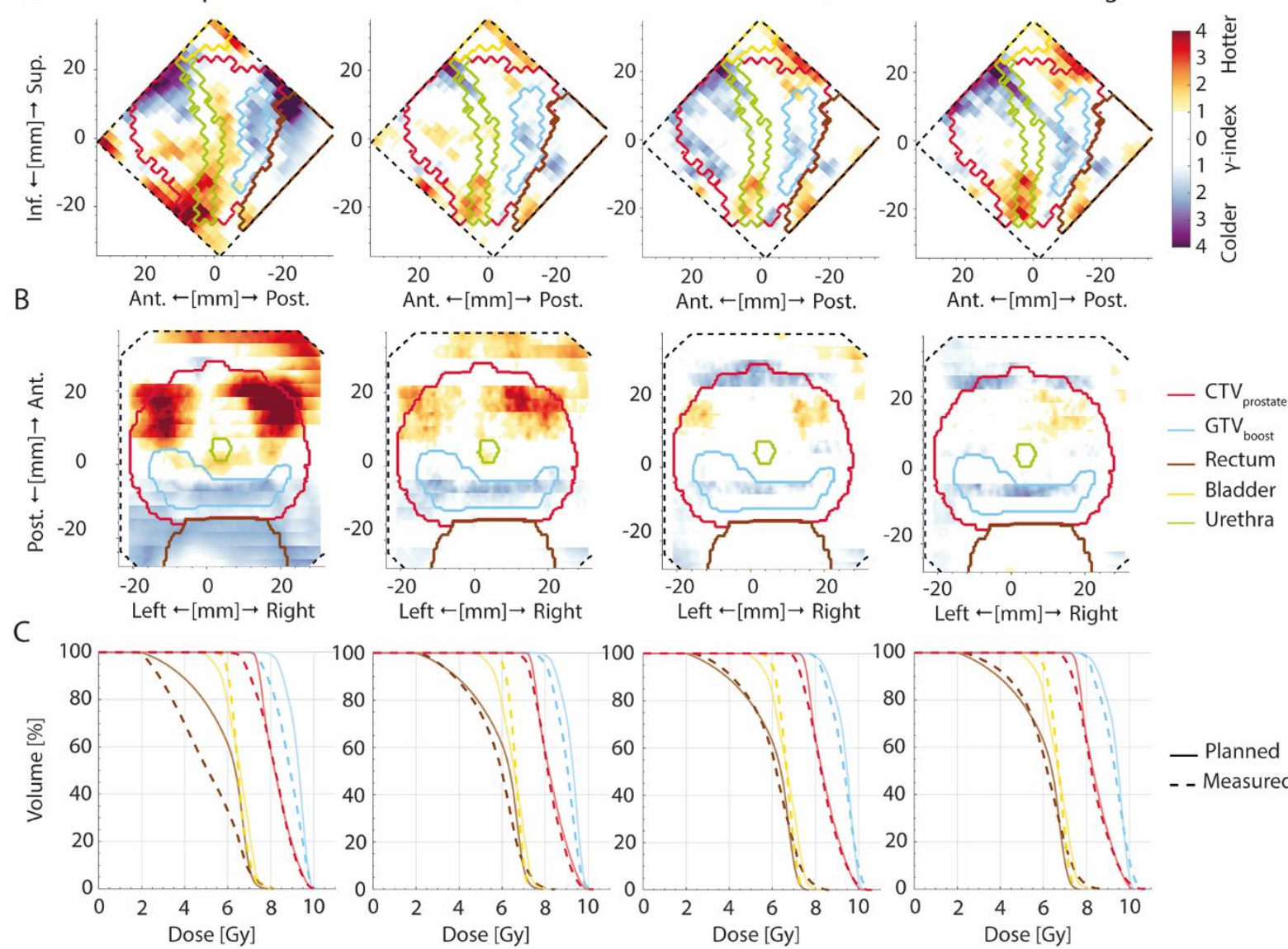

Figure 4. $\boldsymbol{\gamma}$-index map and dose volume histogram for all compensation strategies for the rapid posterior drift. The upper and middle panel depict the $3 \% / 2 \mathrm{~mm} \gamma$-index map of the measured dose with respect to the planned dose when applying a rapid posterior drift (Trace 3 ) during dose delivery without compensation (outer left), with gating (middle left), with MLC tracking (middle right) and with couch tracking (outer right). Points that passed the $3 \% / 2 \mathrm{~mm} \gamma$-index evaluation $(\gamma \leq 1)$ are indicated in white. Points with $\gamma>1$ that measured a higher than planned dose (Hotter) are indicated in a red colourwash whereas points with $\gamma>1$ that measured a lower than planned dose (Cooler) are indicated in a blue colourwash. The volume of interest is depicted by a dashed black line. (A) Sagital slice. (B) Axial slice. (C) Dose volume histogram comparing the measured dose (dashed line) with the planned dose (full line) as reference.

\section{Discussion}

As modern prostate radiotherapy shifts toward steeper dose gradients, smaller treatment margins and fewer treatment fractions, mitigating the deteriorating impact of tumour motion on the delivered dose gains in importance. Both gating and tumour tracking have been shown to mitigate the dosimetric impact of intrafraction translation [13-15]. However, their ability to manage rotation has not yet been thoroughly investigated. In this study, the accuracy of gating, MLC tracking and couch tracking to mitigate the dosimetric impact of intrafraction prostate rotation was investigated experimentally for the first time. 
So far, most performance studies on motion compensation techniques have employed detector arrays mounted on a motion stage whose main advantages are measurement repeatability and motion reproducibility $[21,27,28]$. A limitation of this approach, however, is the limited spatial resolution of the measurement (typically 5-10 mm spacing between individual detectors) and the translation-only motion simulation as the entire detector array is often translated with respect to the beam. Moreover, $3 \mathrm{D}$ dose reconstruction is required to relate the detector array's measurement to the planned dose distribution $[21,28]$. Our approach builds upon previous work by Crijns et al. and uses a dynamic anthropomorphic pelvis phantom that can apply internal motion of a radiochromic film stack [29]. This approach provides high-resolution volumetric measurements of the delivered dose (in-plane resolution of $0.169 \mathrm{~mm}$ and $2.5 \mathrm{~mm}$ slice thickness) comparable to the resolution provided by 3D gel dosimetry (typically $1 \mathrm{~mm}$ grid size) [23,56]. In addition, this design allowed to embed the Calypso transponders directly in the moving film stack and mapped target volume, resulting in an explicit surrogate-to-target relation. Moreover, by using the anthropomorphic phantom for both treatment planning and dose measurements, the impact of the different compensation strategies on the planned dose distribution could be measured directly.

The motion applied by the dynamic phantom was limited to internal lateral rotation of a disk, which contained the film stack. But, since the film stack was located off-center in the disk, this lateral rotation also caused vertical and longitudinal translation of the film stack. This coupled translation and rotation mimics the actual motion of the prostate as multiple studies have demonstrated that motion in the sagittal plane can be approximated by lateral rotations around the apex $[44,45]$. To this end, the prostate contours were mapped onto the film stack such that their apex was located near the rotation point of the disk. It should be noted, however, that due to the geometry of the phantom, the resulting longitudinal translation of the mapped prostate was negatively correlated with its vertical translation, which is opposite to the positive correlation observed in several studies $[10,12,30]$. Nevertheless, given that none of the evaluated compensation techniques made use of a correlation model, this should have no influence on the performance of the techniques. 
Next to the prostate target volumes, the rectum, bladder and urethra contours were rigidly mapped onto the phantom as well. The rectum and bladder were only partially included within the VOI of the film stack (as visualized in Figure 1B) such that the film measurements were limited to the high-dose region only. This is not a major issue, however, as the high-dose regions in the rectum and bladder are more significant predictors of complications compared to low-dose regions [57]. But, it should be noted that the applied internal lateral rotation of the film stack caused the included part of each OAR to rotate together with the prostate. Such a fixed relationship may be assumed for the intraprostatic urethra and to some extent for the anterior rectal wall [58] but is less realistic for the bladder wall. Hence, there may be some uncertainty on the representativity of the measured high-dose region of the mapped bladder contour. Such measurements would ideally be performed using a deformable pelvis phantom [59] due to the deformable nature of the bladder.

Radiochromic films have good spatial resolution, but are prone to noise and inhomogeneities. Lateral scan correction and triple-channel nonuniformity correction were applied to mitigate inhomogeneities and a reference film piece was included in all scans to correct for scan-to-scan and film-to-film response variability $[49,50,60]$. A combined median and Wiener filter was applied to reduce noise while maintaining dose gradients. The resulting uncertainty of film measurements was estimated by repeating the static measurements, which showed a root-mean-square difference below 0.2 Gy within the film stack.

Overall, the use of gating, MLC tracking or couch tracking resulted in better target coverage than no compensation. However, all techniques showed residual motion-related dose errors as indicated by the median $3 \% / 2 \mathrm{~mm} \gamma$-index agreement for the prostate CTV of $69.3 \%$ with gating, $66.6 \%$ with MLC tracking and $71.0 \%$ with couch tracking. This is in contrast to Ehrbar et al. who found a median $2 \% / 2 \mathrm{~mm} \gamma$-index agreement of $98.6 \%$ with MLC tracking and $100.0 \%$ with couch tracking compared to $83.9 \%$ without compensation [21]. Similarly, Hansen et al. observed a decrease of the $2 \% / 2 \mathrm{~mm} \gamma^{-}$ index failure rates from median $21.6 \%$ without tracking to $0.7 \%$ with MLC tracking and $0.0 \%$ with couch tracking [27]. However, both studies assumed that the recorded prostate motion was purely 
translational and thus applied only translation during dose delivery compared to the coupled rotation and translation that was applied in this work. This suggests that the residual motion-related dose errors are caused by a lack of rotation correction in the investigated techniques.

Both MLC tracking and couch tracking used the Calypso system to monitor the 3D target centroid position and transfer this signal to the iTools Tracking platform to adapt the MLC or couch position, thus omitting rotations. Gating, on the other hand, monitors the displacement of each marker individually, which may allow it to detect gross rotation as markers located at the base show larger displacements than those located at the apex $[33,44]$. However, since the maximum lateral rotation correction of the treatment couch are often limited to $3^{\circ}-5^{\circ}[46,61]$, residual rotations will remain, thus preventing full rotational compensation through counter-rotation of the couch. In addition, such rotational compensations may increase the uncertainty of the body position relative to the couch due to gravity [62].

In practice, prostate translation will be a combination of both pure translation and translation caused by rotation around its apex. As the investigated techniques have only limited rotational compensation, our experiments effectively modelled the worst-case scenario for these compensation techniques since the applied translation was purely of rotational nature. But, since gating and tracking have been shown to mitigate the dosimetric impact of translations almost completely [13-15], including pure translations into the experiments would result in only a negligible increase of residual motion-related dose errors on top of those caused by the remaining uncorrected rotations.

This work focused on the performance of gating, MLC tracking and couch tracking implemented on standard clinical C-arm linacs, which all have limited rotational compensation. Other compensation techniques may allow to correct for intrafraction rotation, but these were not part of this work as these required dedicated linac systems or are not yet fully implemented. Robotic tracking, for instance, combines a robotic treatment couch with a linac mounted on a robotic manipulator, allowing it to correct for intrafraction rotation up to $10^{\circ}$ [63]. Rotational compensation through the MLC has been proposed for both IMRT $[31,64,65]$ and VMAT $[66]$ but is not yet available. 
Estimating the clinical impact of the residual motion-related dose errors on tumour control probability (TCP) is difficult as application of the TCP models constructed for the conventional fractionated regimen to ultrahypofractionated treatments may not be assumed without verification [67]. Moreover, the performed experiments emulate only a single treatment fraction such that the relative magnitude of the residual dose error may decrease over an entire treatment course.

Nonetheless, the residual motion-related dose errors observed in this study indicate a need to correct for intrafraction prostate rotation and advocate for the implementation of rotational compensation into current compensation techniques, such as MLC tracking. The dynamic pelvis phantom and film stack used in this work may offer a valuable quality assurance tool for the dosimetric validation of such new compensation techniques.

\section{Conclusion}

Compensation of intrafraction prostate rotation with gating, MLC tracking and couch tracking was investigated experimentally for the first time. All three techniques improved the dosimetric accuracy, but residual motion-related dose errors remained due to the lack of rotation correction.

\section{Acknowledgements}

Robin De Roover is funded by a Kom op tegen Kanker (Stand up to Cancer) grant from the Flemish Cancer Society. This work was supported by a travel grant from the research foundation Flanders (FWO). This work was partly supported by Varian Medical Systems. 


\section{References}

1. Mahase SS, Angelo DD, Kang J, Hu JC, Barbieri CE, Nagar H. Trends in the Use of Stereotactic Body Radiotherapy for Treatment of Prostate Cancer in the United States. JAMA Netw Open. 2020;3(2):e1920471.

2. Kishan AU, Dang A, Katz AJ, Mantz CA, Collins SP, Aghdam N, et al. Long-term Outcomes of Stereotactic Body Radiotherapy for Low-Risk and Intermediate-Risk Prostate Cancer. JAMA Netw Open. 2019;2(2):1-13.

3. Jackson WC, Silva J, Hartman HE, Dess RT, Kishan AU, Beeler WH, et al. Stereotactic Body Radiotherapy for Localized Prostate Cancer: A Systematic Review and MetaAnalysis of Over 6,000 Patients Treated On Prospective Studies. Int J Radiat Oncol. 2019;104(4):778-89.

4. Lehrer EJ, Kishan AU, Yu JB, Trifiletti DM, Showalter TN, Ellis $R$, et al. Ultrahypofractionated vs hypofractionated and conventionally fractionated radiation therapy for localized prostate cancer: a systematic review and meta-analysis of phase III randomized trials. Radiother Oncol. 2020;148:235-42.

5. Widmark A, Gunnlaugsson A, Beckman L, Thellenberg-karlsson C, Hoyer M, Lagerlund $M$, et al. Ultra-hypofractionated versus conventionally fractionated radiotherapy for prostate cancer: 5-year outcomes of the HYPO-RT-PC randomised, non-inferiority, phase 3 trial. Lancet. 2019;6736(19):1-11.

6. Brand DH, Tree AC, Ostler P, Voet H Van Der, Loblaw A, Chu W, et al. Intensitymodulated fractionated radiotherapy versus stereotactic body radiotherapy for prostate cancer (PACE-B): acute toxicity findings from an international, randomised, open-label , phase 3, non-inferiority trial. Lancet Oncol. 2019;2045(19):1-13.

390 7. Zietman AL. Making radiation therapy for prostate cancer more economical and more convenient. J Clin Oncol. 2016;34(20):2323-4.

8. Benedict SH, Followill D, Galvin JM, Hinson W, Kavanagh B, Keall P, et al. Stereotactic body radiation therapy: The report of AAPM Task Group 101. Med Phys. 2010;37(8):4078-101.

9. Morgan SC, Hoffman K, Loblaw DA, Buyyounouski MK, Patton C, Barocas D, et al. Hypofractionated Radiation Therapy for Localized Prostate Cancer: Executive Summary of an ASTRO, ASCO and AUA Evidence-Based Guideline. J Clin Oncol. 2018;36(34):341130.

10. Kupelian P, Willoughby $T$, Mahadevan A, Djemil T, Weinstein G, Jani $S$, et al. Multiinstitutional clinical experience with the Calypso System in localization and continuous, real-time monitoring of the prostate gland during external radiotherapy. Int J Radiat Oncol Biol Phys. 2007;67(4):1088-98.

11. Langen KM, Willoughby TR, Meeks SL, Santhanam A, Cunningham A, Levine L, et al. Observations on Real-Time Prostate Gland Motion Using Electromagnetic Tracking. Int J Radiat Oncol Biol Phys. 2008;71(4):1084-90.

12. Ng JA, Booth JT, Poulsen PR, Fledelius W, Worm ES, Eade T, et al. Kilovoltage intrafraction monitoring for prostate intensity modulated arc therapy: First clinical results. Int J Radiat Oncol Biol Phys. 2012;84(5):e655--e661.

13. Colvill E, Poulsen PR, Booth JT, O'Brien RT, Ng JA, Keall PJ. DMLC tracking and gating can improve dose coverage for prostate VMAT. Med Phys. 2014;41(9):91705.

14. Colvill E, Booth JT, Brien RTO, Eade TN, Kneebone AB, Poulsen PR, et al. Multileaf Collimator Tracking Improves Dose Delivery for Prostate Cancer Radiation Therapy : 
Results of the First Clinical Trial. Radiat Oncol Biol. 2015;92(5):1141-7.

415

420

15. Keall P, Nguyen DT, Brien RO, Hewson E, Ball H, Poulsen P, et al. Real-Time ImageGuided Ablative Prostate Cancer Radiation Therapy: Results from the TROG 15.01 SPARK Trial. Int J Radiat Oncol • Biol • Phys. 2020;

16. Lovelock DM, Messineo AP, Cox BW, Kollmeier MA, Zelefsky MJ. Continuous monitoring and intrafraction target position correction during treatment improves target coverage for patients undergoing sbrt prostate therapy. Int J Radiat Oncol Biol Phys. 2015;91(3):588-94.

17. Keall PJ, Ng JA, Juneja P, O'Brien RT, Huang CY, Colvill E, et al. Real-Time 3D Image Guidance Using a Standard LINAC: Measured Motion, Accuracy, and Precision of the First Prospective Clinical Trial of Kilovoltage Intrafraction Monitoring-Guided Gating for Prostate Cancer Radiation Therapy. Int J Radiat Oncol Biol Phys. 2016;94(5):1015-21.

18. Rosario T, van der Weide L, Admiraal M, Piet M, Slotman B, Cuijpers J. Toward planning target volume margin reduction for the prostate using intrafraction motion correction with online kV imaging and automatic detection of implanted gold seeds. Pract Radiat Oncol. 2018;

19. Kisivan K, Antal G, Gulyban A, Glavak C, Laszlo Z, Kalincsak J, et al. Triggered Imaging with Auto Beam Hold and pre-/post-treatment $\mathrm{CBCT}$ during prostate SABR: analysis of time efficiency, target coverage and normal volume changes. Pract Radiat Oncol. 2020;

20. Keall P., Colvill E, O'Brien R, Ng JA, Poulsen PR, Eade T, et al. The first clinical implementation of electromagnetic transponder-guided MLC tracking. Med Phys. 2014;41(2):020702.

21. Ehrbar S, Schmid S, Jöhl A, Klöck S, Guckenberger M, Riesterer O, et al. Comparison of multi-leaf collimator tracking and treatment-couch tracking during stereotactic body radiation therapy of prostate cancer. Radiother Oncol. 2017;125(3):445-52.

22. Keall PJ, Nguyen DT, O'Brien R, Caillet V, Hewson E, Poulsen PR, et al. The first clinical implementation of real-time image-guided adaptive radiotherapy using a standard linear accelerator. Radiother Oncol. 2018;1-6.

23. Ceberg S, Falk M, Af Rosenschöld PM, Cattell H, Gustafsson H, Keall P, et al. Tumortracking radiotherapy of moving targets; verification using 3D polymer gel, 2D ionchamber array and biplanar diode array. J Phys Conf Ser. 2010;250:235-9.

24. Keall PJ, Sawant A, Cho B, Ruan D, Wu J, Poulsen P, et al. Electromagnetic-guided dynamic multileaf collimator tracking enables motion management for intensitymodulated arc therapy. Int J Radiat Oncol Biol Phys. 2011;79(1):312-20.

25. Poulsen PR, Fledelius W, Cho B, Keall P. Image-based dynamic multileaf collimator tracking of moving targets during intensity-modulated arc therapy. Int J Radiat Oncol Biol Phys. 2012;83(2):e265-71.

26. Colvill E, Booth J, Nill S, Fast M, Bedford J, Oelfke U, et al. A dosimetric comparison of real-time adaptive and non-adaptive radiotherapy: $A$ multi-institutional study encompassing robotic, gimbaled, multileaf collimator and couch tracking. Radiother Oncol. 2016;119(1):159-65.

27. Hansen R, Ravkilde T, Worm ES, Toftegaard J, Grau C, Macek K, et al. Electromagnetic guided couch and multileaf collimator tracking on a TrueBeam accelerator. Med Phys. 2016;43(5):2387-98.

28. Ehrbar S, Schmid S, Johl A, Klock S, Guckenberger M, Riesterer O, et al. Validation of dynamic treatment-couch tracking for prostate SBRT. Med Phys. 2017;44(6):2466-77.

29. Crijns W, Defraene G, Van Herck H, Depuydt T, Haustermans K, Maes F, et al. Potential 
benefits of dosimetric VMAT tracking verified with 3D film measurements. Med Phys. 2016;43(5):2162-73.

30. Huang CY, Tehrani JN, Ng JA, Booth J, Keall P. Six degrees-of-freedom prostate and lung tumor motion measurements using kilovoltage intrafraction monitoring. Int J Radiat Oncol Biol Phys. 2015;91(2):368-75.

31. Deutschmann H, Kametriser $G$, Steininger $P$, Scherer $P$, Schöller H, Gaisberger $C$, et al. First clinical release of an online, adaptive, aperture-based image-guided radiotherapy strategy in intensity-modulated radiotherapy to correct for inter- and intrafractional rotations of the prostate. Int J Radiat Oncol Biol Phys. 2012;83(5):1624-32.

32. Rijkhorst EJ, Lakeman A, Nijkamp J, De Bois J, van Herk M, Lebesque J V., et al. Strategies for online organ motion correction for intensity-modulated radiotherapy of prostate cancer: prostate, rectum and bladder dose effects. Int J Radiat Oncol Biol Phys. 2009;75(4):1254-60.

33. Amro H, Hamstra DA, McShan DL, Sandler H, Vineberg K, Hadley S, et al. The dosimetric impact of prostate rotations during electromagnetically guided external-beam radiation therapy. Int J Radiat Oncol Biol Phys. 2013;85(1):230-6.

34. Wolf J, Nicholls J, Hunter P, Nguyen DT, Keall P, Martin J. Dosimetric impact of intrafraction rotations in stereotactic prostate radiotherapy: A subset analysis of the TROG 15.01 SPARK trial. Radiother Oncol. 2019;136:143-7.

35. Lips IM, van der Heide UA, Haustermans K, van Lin ENJT, Pos F, Franken SPG, et al. Single blind randomized Phase III trial to investigate the benefit of a focal lesion ablative microboost in prostate cancer (FLAME-trial): Study protocol for a randomized controlled trial. Trials. 2011;12(December).

36. Salembier C, Villeirs G, De Bari B, Hoskin P, Pieters BR, Van Vulpen $M$, et al. ESTRO ACROP consensus guideline on CT- and MRI-based target volume delineation for primary radiation therapy of localized prostate cancer. Radiother Oncol. 2018;d:1-13.

37. Dinh CV, Steenbergen P, Ghobadi G, van der Poel H, Heijmink SWTPJ, de Jong J, et al. Multi-center validation of prostate tumor localization using multi-parametric MRI and prior knowledge. Med Phys. 2016;

38. van Schie MA, Dinh C V., Houdt PJ va., Pos FJ, Heijmink SWTJP, Kerkmeijer LGW, et al. Contouring of prostate tumors on multiparametric MRI: Evaluation of clinical delineations in a multicenter radiotherapy trial. Radiother Oncol. 2018;128(2):321-6.

39. Lieberfarb ME, Schultz D, Whittington R, Malkowicz B, Tomaszewski JE, Weinstein M, et al. Using PSA, biopsy Gleason score, clinical stage, and the percentage of positive biopsies to identify optimal candidates for prostate-only radiation therapy. Int J Radiat Oncol. 2002;53(4):898-903.

40. Gay HA, Barthold HJ, O'Meara E, Bosch WR, El Naqa I, Al-Lozi R, et al. Pelvic normal tissue contouring guidelines for radiation therapy: a Radiation Therapy Oncology Group consensus panel atlas. Int J Radiat Oncol Biol Phys. 2012;83(3):e353-62.

41. Draulans C, De Roover R, van der Heide UA, Haustermans K, Pos F, Smeenk RJ, et al. Stereotactic body radiation therapy with optional focal lesion ablative microboost in prostate cancer: Topical review and multicenter consensus. Radiother Oncol. 2019;140:131-42.

42. Draulans C, Heide UA Van Der, Haustermans K, Pos FJ, Voort J Van Der, Zyp V, et al. Primary endpoint analysis of the multicentre phase II hypo-FLAME trial for intermediate and high risk prostate cancer. Radiother Oncol. 2020;147:92-8.

43. Ghadjar P, Fiorino $C$, Munck af Rosenschöld P, Pinkawa M, Zilli T, van der Heide UA. 
ESTRO ACROP consensus guideline on the use of image guided radiation therapy for localized prostate cancer. Radiother Oncol. 2019;141:5-13.

44. de Boer HCJ, van Os MJH, Jansen PP, Heijmen BJM. Application of the No Action Level (NAL) protocol to correct for prostate motion based on electronic portal imaging of implanted markers. Int J Radiat Oncol Biol Phys. 2005;61(4):969-83.

45. Boda-Heggemann J, Köhler F, Wertz H, Welzel G, Riesenacker N, Schäfer J, et al. Fiducialbased quantification of prostate tilt using cone beam computer tomography (CBCT). Radiother Oncol. 2007;85(2):247-50.

515 46. Schmidhalter D, Fix MK, Wyss M, Schaer N, Munro P, Scheib S, et al. Evaluation of a new six degrees of freedom couch for radiation therapy. Med Phys. 2013;40(11):1-11.

47. Sharp GC, Jiang SB, Shimizu S, Shirato H. Prediction of respiratory tumour motion for real-time image-guided radiotherapy. Phys Med Biol. 2004;49(3):425-40.

48. Crijns W, Maes F, van der Heide $U$ a, Van den Heuvel F. Calibrating page sized Gafchromic EBT3 films. Med Phys. 2013;40(1):012102.

49. Micke A, Lewis DF, Yu X. Multichannel film dosimetry with nonuniformity correction. Med Phys. 2011;38(5):2523-34.

50. Lewis D, Devic S. Correcting scan-to-scan response variability for a radiochromic filmbased reference dosimetry system. Med Phys. 2015;42(10):5692-701.

51. Pinter $C$, Lasso A, Wang A, Jaffray $D$, Fichtinger $G$. SlicerRT: radiation therapy research toolkit for 3D Slicer. Med Phys. 2012;39(10):6332-8.

52. Low D a., Harms WB, Mutic S, Purdy J a. A technique for the quantitative evaluation of dose distributions. Med Phys. 1998;25(5):656-61.

53. Depuydt T, Van Esch A, Huyskens DP. A quantitative evaluation of IMRT dose distributions: Refinement and clinical assessment of the gamma evaluation. Radiother Oncol. 2002;62(3):309-19.

54. Miften M, Olch A, Mihailidis D, Moran J, Pawlicki T, Molineu A, et al. Tolerance limits and methodologies for IMRT measurement-based verification QA: Recommendations of AAPM Task Group No. 218. Med Phys. 2018;45(4):e53-83.

55. Benjamini Y, Hochberg Y. Controlling the False Discovery Rate: a Practical and Powerful Approach to Multiple Testing. J R Stat Soc B. 1995;57(1):289-300.

56. Skyt PS, Petersen JBB, Yates ES, Poulsen PR, Ravkilde TL, Balling P, et al. Dosimetric verification of complex radiotherapy with a 3D optically based dosimetry system: Dose painting and target tracking. Acta Oncol (Madr). 2013;52(7):1445-50.

57. Alayed Y, Davidson M, Quon H, Cheung P, Chu W, Chung HT, et al. Dosimetric predictors of toxicity and quality of life following prostate stereotactic ablative radiotherapy. Radiother Oncol. 2020;144:135-40.

58. Padhani AR, Khoo VS, Suckling J, Husband JE, Leach MO, Dearnaley DP. Evaluating the effect of rectal distension and rectal movement on prostate gland position using cine MRI. Int J Radiat Oncol Biol Phys. 1999;44(3):525-33.

59. Bohoudi O, Lagerwaard FJ, Bruynzeel AME, Niebuhr NI, Johnen W, Senan S, et al. Endto-end empirical validation of dose accumulation in MRI-guided adaptive radiotherapy for prostate cancer using an anthropomorphic deformable pelvis phantom. Radiother Oncol. 2019;(xxxx).

550 60. Crijns W, Maes F, van der Heide U a, Van den Heuvel F. Calibrating page sized Gafchromic EBT3 films. Med Phys. 2013;40(1):12102.

61. Kilby W, Dooley JR, Kuduvalli G, Sayeh S, Maurer CR. The CyberKnife ${ }^{\circledR}$ robotic radiosurgery system in 2010. Technol Cancer Res Treat. 2010;9(5):433-52. 
62. Jöhl A, Bogowicz M, Ehrbar S, Guckenberger M, Klöck S, Meboldt M, et al. Body motion during dynamic couch tracking with healthy volunteers. 2010;1-25.

63. Van De Water S, Valli L, Aluwini S, Lanconelli N, Heijmen B, Hoogeman M. Intrafraction prostate translations and rotations during hypofractionated robotic radiation surgery: Dosimetric impact of correction strategies and margins. Int J Radiat Oncol Biol Phys. 2014;

560 64. Wu J, Ruan D, Cho B, Sawant A, Petersen J, Newell U, et al. Electromagnetic detection and real-time DMLC adaptation to target rotation during radiotherapy. Int J Radiat Oncol Biol Phys. 2012;82(3):e545-53.

65. Crijns W, Van Herck H, Defraene G, Van Den Bergh L, Slagmolen P, Haustermans K, et al. Dosimetric adaptive IMRT driven by fiducial points. Med Phys. 2014;41(6).

66. Crijns W, Defraene G, Van Herck H, Depuydt T, Haustermans K, Maes F, et al. Online adaptation and verification of VMAT. Med Phys. 2015;42(7):3877-91.

67. Vogelius IR, Bentzen SM. Diminishing returns from ultra-hypofractionated radiation therapy for prostate cancer. Int J Radiat Oncol. 2020; 